\title{
EVALUATING THREE INTERFACE TECHNOLOGIES IN ASSISTING PEDESTRIANS' SPATIAL KNOWLEDGE ACQUISITION
}

\author{
H. Huang*, M. Schmidt, G. Gartner \\ Institute of Geoinformation and Cartography, Vienna University of Technology, Vienna - \\ \{haosheng.huang, manuela.schmidt, georg.gartner\}@tuwien.ac.at
}

KEY WORDS: Comparison, GPS/INS, Visualization, Mobile, Experimental, User, Performance

\begin{abstract}
:
Recent years have seen raising interests in mobile pedestrian navigation systems. Different interface technologies can be used to communicate/convey route directions to pedestrians, such as mobile maps, voices, and augmented reality (AR). Many field experiments have been conducted to study the effectiveness of different interface technologies in guiding pedestrians to their destinations. In contrast to other field studies, this article aims at investigating the influence of different interface technologies on spatial knowledge acquisition (spatial learning). With sufficient spatial knowledge about an environment, people can still find their way when navigation systems fail (e.g. out of battery).

The goal of this article is to empirically evaluate three GPS-based navigation prototypes (implementing mobile map-based, ARbased, and voice-based guidance respectively) in supporting spatial knowledge acquisition. The field test showed that in terms of spatial knowledge acquisition, the three interface technologies led to comparable poor results, which were also not significantly different from each other. This article concludes with some implications for designing mobile pedestrian navigation systems.
\end{abstract}

\section{INTRODUCTION}

In recent years, interest in using mobile phones to assist pedestrian wayfinding in unfamiliar environments is increasing. Mobile pedestrian navigation systems are designed for this purpose. Different interface technologies can be used to communicate/convey route information to pedestrians, such as mobile maps, voice, 3D, images and augmented reality (AR).

There are many field tests studying the effectiveness of different interface technologies in supporting pedestrian navigation (Rehrl et al., 2010; Walther-Franks, 2007), mainly focusing on how many errors they made during wayfinding, and how much time they took to finish the route. In contrast to them, this article is interested in studying the influence of different interface technologies on spatial knowledge acquisition. With sufficient spatial knowledge about an environment, people can still find their way when navigation systems fail (e.g. out of battery). Therefore, it is important to investigate how navigation systems affect the acquisition of spatial knowledge during navigation.

There are some studies empirically investigating the acquisition of spatial knowledge in the context of pedestrian navigation. Gartner and Hiller (2009) investigated maps with different display sizes and showed that display size influenced spatial knowledge acquisition during navigation. Münzer et al. (2006) empirically compared paper maps with three electronic navigation systems, and found that navigation system users showed good route knowledge and poor survey knowledge, in contrast, paper map users showed better survey knowledge and nearly perfect route knowledge. It is important to note that most of the above studies employed the "Wizard of Oz" prototyping (e.g. without using the GPS) (Wikipedia, 2011). In contrast, Ishikawa et al. (2008) compared the acquisition of spatial knowledge with a map-based GPS navigation system, paper maps and direct experience of travelling, and showed a poorer performance by subjects using the GPS-based system.

In this article, we will report a field test, focusing on comparing the influence of mobile maps, AR, and voice on spatial knowledge acquisition in the context of GPS-based pedestrian navigation. Three navigation prototypes, implementing mobile map-based, AR-based, and voice-based guidance respectively, were developed based on recent findings in literature. Subjects were asked to use the interfaces to solve some real-world navigation tasks in the city centre of Salzburg (Austria).

The rest of this article is structured as follows. In section 2, the three navigation prototypes are implemented by integrating recent findings on conveying route information. The study design is presented in section 3. Section 4 analyses and discusses the results. Finally, we draw conclusions and present future work.

\section{CONVEYING ROUTE INFORMATION WITH MOBILE MAPS, AR AND VOICE}

For studying spatial knowledge acquisition with different interface technologies, we used three self-implemented mobile navigation systems running on Apple's iPhone 4. These systems used map-based, AR-based, and voice-based interfaces respectively. Recent findings on pedestrian navigation from literature, such as Tversky (1992), Tversky and Lee (1999), and Agrawala (2001), were integrated and considered when developing these systems.

Figures 1 and 2 show the screenshots for map-based and ARbased interface. A detailed description on these interfaces is presented in Huang et al. (2012).

\footnotetext{
* Corresponding author.
} 


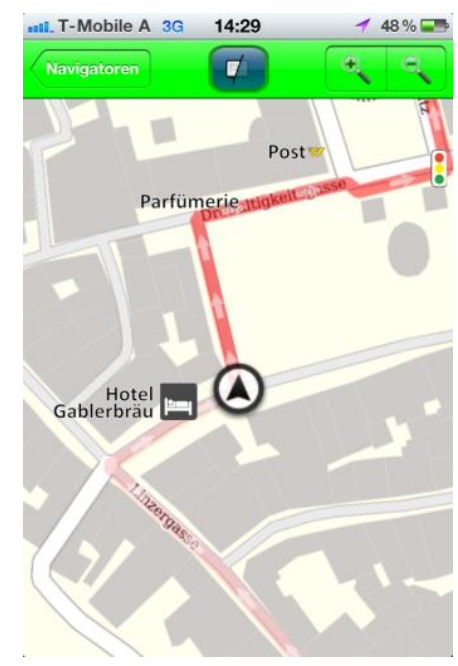

Figure 1. A screenshot of the map-based interface, with an egocentric view, distinction between the past and future paths, automatic adaptation to real-time location, zooming and panning functions, etc (@) Salzburg Research, Map data: OpenStreetMap and Contributors, CC-BY-SA).

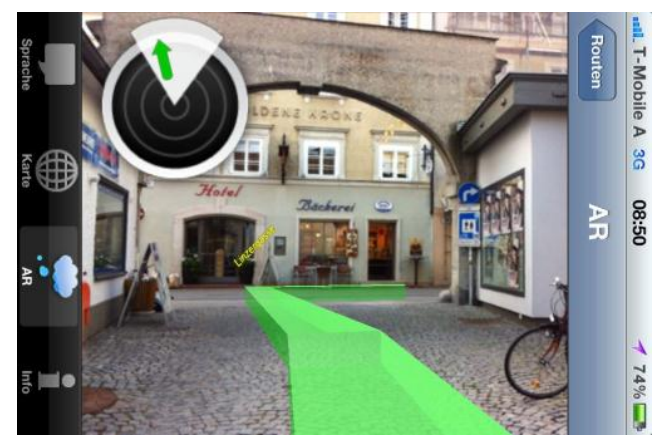

Figure 2. A screenshot of the AR-based interface, with a real world camera view, route overlay, street names and relevant landmarks (@ Salzburg Research).

The development of the voice-based interface was based on the findings of the previous project SemWay, in which a formal model of navigation language was designed (Rehrl et al. 2009). With the model, semantic-based route instructions instead of metric-based instructions can be provided, for example, "walk straight, pass the theatre, and walk to the crossing" instead of "walk straight for $103 \mathrm{~m}$ ". When a user gets close to a decision point, the mobile device vibrates, and plays the voice instruction describing the actions from this decision point to the next.

\section{STUDY DESIGN}

A route in the city centre of Salzburg was selected for the test. It was divided into three sub-routes, each with 9 decision points (i.e. interactions where multiple outgoing choices exist).

Three tasks are designed to assess their acquisition of spatial knowledge:

1. Landmark recognition task: Given a photo album, participants were asked to identify all pictures that they thought were along the route just walked.
2. Route direction task: For the chosen pictures that were at intersections, participants should indicate the turn they had taken at each of them.

3. Landmark placement task: Participants were asked to place the chosen pictures on an OpenStreetMap map of the current area.

Twenty four participants took part in the study (12 female and 12 male). The mean age was about 42 years (range 21-73). They were randomly divided into three groups, each with 8 participants (4 female and 4 male). A within-subject design and a counterbalancing consideration were used for the test, i.e. for each sub-route, these three groups each used one of the navigation prototypes. When they reached the next sub-route, they switched to another prototype.

After a brief training session, the participants were led to the starting point of the first sub-route. Their tasks were to navigate to the end of the sub-route. If participants decided wrongly at a decision point, the observing researcher used gestures to indicate the correct choice. No other assistance was given during navigation. When reaching the end of the sub-route, participants were asked to answer questionnaires assessing usability and task load. In addition, they were asked to do the following tasks one by one: indicating their familiarity with the current sub-route before the test, landmark recognition task, route direction task, and landmark placement task. None of the tasks had a time limit for answering. Only accuracy performance was measured. When finishing all these tasks, participants switched to another prototype, and the same procedure was repeated for the next sub-route. Each test was completed within 1.5 hours in total.

\section{RESULTS AND DISCUSSION}

The field experiment was completed in July 2011. All participants successfully completed the navigation tasks. The results of the experiment included two parts: wayfinding performance and user experience, and spatial knowledge acquisition. In this article, we report the results of spatial knowledge acquisition. We only considered the results from participants who were unfamiliar with the sub-routes. In total, we had 32 participant/sub-route pairs (10 for mobile maps, 13 for $\mathrm{AR}$, and 9 for voice). The male-female ratios were similar in the three interface technologies. Figure 3 shows the results.

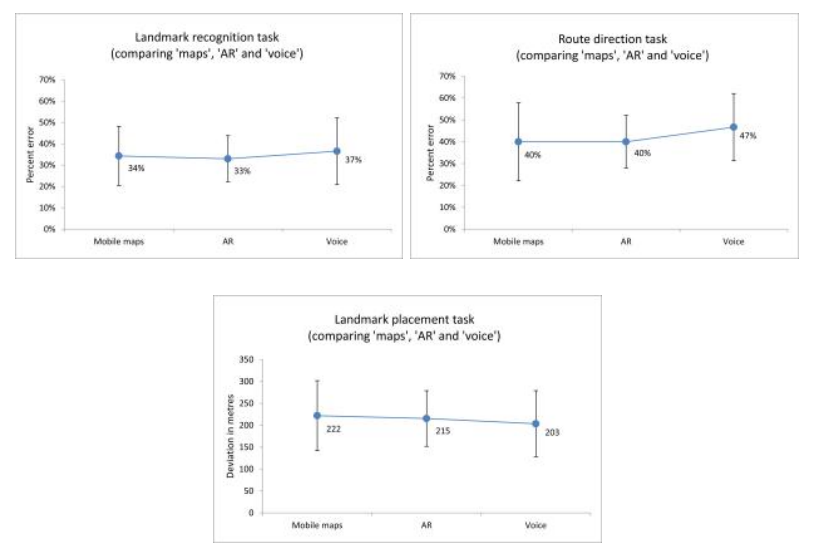

Figure 3. Comparison of the influence of mobile maps, AR, and voice on spatial knowledge acquisition: the landmark recognition task (mean percentage error), 
the route direction task (mean percentage error), and the landmark placement task (mean deviation in metres). Vertical bars denote $95 \%$ confidence intervals.

Results in Figure 3 show that, for all three tasks, the differences among the three interface technologies were not significant at the 5\% level. It is also worth noting that, in all three interface technologies, participants did not perform so well in either of the three tasks (about 33\%-47\% errors in the first two tasks, about $42 \%-46 \%$ of the route length in the last task).

These non-significant results are consistent with the findings of Münzer et al. (2006), in which they compared paper maps with three electronic navigation systems, and showed that the three navigation systems did not lead to significant differences in spatial knowledge acquisition.

One of the possible interpretations of the above results can be the effect of "passive navigation". The design of all of the three navigation prototypes was optimized to make navigation as easy as possible, for example, with the help of GPS, users were free from the mental efforts of continuously maintaining where they were. In addition, for all three interface technologies, the mental spatial transformation was avoided and not needed anymore with the help of the employed egocentric frame of reference. In short, for all three interface technologies, participants did not need many active mental efforts to derive the direction information. As spatial learning is an effortful process (Aginsky and Rensink, 1997; Münzer et al., 2006; Parush et al., 2007), all three interface technologies led to poor results in spatial knowledge acquisition, which were also not significant from each other.

\section{CONCLUSIONS AND FUTURE WORK}

More and more people are relying on pedestrian navigation systems to find ways in unfamiliar environments. Therefore, it is essential to study how these systems affect the acquisition of spatial knowledge, which is required when navigation systems fail (e.g. out of battery). This article studied the influence of different interface technologies (mobile maps, AR, and voice) on spatial knowledge acquisition in a field test in an urban environment. The field test showed that in terms of spatial knowledge acquisition, the difference among the three interface technologies was not significant. Some possible interpretations of the results were discussed.

We are aware that the number of participants were comparably small. Therefore, currently, we are planning another experiment with more participants involved. With this, we aim to have a more in-depth analysis of the influence of different interface technologies on spatial knowledge acquisition. More importantly, we are interested in designing a navigation system facilitating both wayfinding and spatial knowledge acquisition.

\section{REFERENCES}

Aginsky, V., Harris, C., Rensink, R. and Beusmans, J., 1997. Two strategies for learning a route in a driving simulator. Journal of Environmental Psychology, 17, pp. 317-331.

Agrawala, M., 2001. Visualizing Route Maps. PhD thesis, Stanford University.
Gartner, G. and Hiller, W., 2009. Impact of restricted display size on spatial knowledge acquisition in the context of pedestrian navigation. In: Location Based Services and TeleCartography II, ed. by Gartner, G. and Rehrl, K., pp. 155166 , Springer.

Huang, H., Schmidt, M. and Gartner, G., 2012. Spatial Knowledge Acquisition with Mobile Maps, Augmented Reality and Voice in the Context of GPS-based Pedestrian Navigation: Results from a Field Test. Accepted for Cartography and Geographic Information Science.

Ishikawa, T., Fujiwara, H. Imai, O. and Okabe, A., 2008. Wayfinding with a GPS-based mobile navigation system: a comparison with maps and direct experience. Journal of Environmental Psychology, 28(1), pp.74-82.

Münzer, S., Zimmer, H., Schwalm, M., Baus, J. and Aslan, I., 2006. Computer Assisted Navigation and the Acquisition of Route and Survey Knowledge. Journal of Environmental Psychology, 26, pp. 300-308.

Parush, A., Ahuvia, S. and Erev, I., 2007. Degradation in spatial knowledge acquisition when using automatic navigation systems. In: COSIT 2007, ed. by Winter, S., pp. 238-254, Springer.

Rehrl, K., Häusler, E. and Leitinger, S., 2010. Comparing the effectiveness of GPS-enhanced voice guidance for pedestrians with metric- and landmark-based instruction sets. In: GIScience 2010, ed. by Fabrikant, S.I., Reichenbacher, T., van Kreveld, M. and Schlieder, C., pp. 189-203, Springer.

Rehrl, K., Leitinger, S., Gartner, G. and Ortag, F., 2009. An analysis of direction and motion concepts in verbal descriptions of route choices. In: COSIT 2009, ed. by Stewart Hornsby, K., Claramunt, C., Denis, M. and Ligozat, G. pp. 471-488, Springer.

Tversky, B., 1992. Distortions in cognitive maps. Geoforum, 23(2), pp. 131-138.

Tversky, B. and Lee, P., 1999. Pictorial and verbal tools for conveying routes. In: COSIT 1999, ed. by Freska, C. and Mark, D., pp. 51-64, Springer.

Walther-Franks, B., 2007. Augmented reality on handhelds for pedestrian navigation, Master thesis, University of Bremen.

Wikipedia, 2011. Wizard of $\mathrm{Oz}$ experiment. http://en.wikipedia.org/wiki/Wizard_of_Oz_experiment (31 March 2012).

\section{ACKNOWLEDGMENTS}

This work was supported by the ways 2 navigate project, funded by the Austria FFG's ways2go program (2009). We thank our partners Salzburg Research, TraffiCon, WalkSpaceMobilität, and FACTUM for their contributions to the project. 